\title{
Visibilización de dificultades educativas: Los retos del gobierno colombiano y de las comunidades educativas para afrontarlas
}

Visibility of educational difficulties: the challenges of the Colombian government and educational communities to face them

\section{Luz Stella Montero Mahecha}

\section{Citar este documento según modelo APA}

Montero Mahecha, Luz Stella. (2021). VIsibilización de dificultades educativas: Los retos del gobierno colombiano y de las comunidades educativas para afrontarlas. Revista Actualidades Investigativas en Educación, 21(3), 1-24. Doi. 10.15517/aie.v21i2.46489 


\title{
Visibilización de dificultades educativas: Los retos del gobierno colombiano y de las comunidades educativas para afrontarlas Visibility of educational difficulties: the challenges of the Colombian government and educational communities to face them
}

\section{Luz Stella Montero Mahecha ${ }^{1}$}

\begin{abstract}
Resumen: El presente ensayo se realiza con el fin de dar a conocer los obstáculos que ha afrontado la educación pública colombiana y la inequidad que se ha visibilizado con la llegada de la pandemia del Covid-19. Para ello se analizan diversas dificultades, entre las que se encuentran, la carencia de un Proyecto Educativo Institucional sólido, la ausencia de conectividad y la insuficiencia de equipos y conocimientos tecnológicos de la comunidad educativa, los cuales tienen de trasfondo la falta de conocimiento en el manejo de herramientas digitales y/o la escasez de recursos económicos, al igual que la carencia de hábitos de auto aprendizaje. De allí que es necesario que quienes estén involucrados en el proceso enseñanza aprendizaje, desempeñen con compromiso el papel que les corresponde, de tal manera que se cumpla a cabalidad con el currículo, independientemente de las circunstancias que lo rodeen. Como conclusión, se establece la responsabilidad que debe asumir el Estado en la asignación de presupuesto para el sector educativo y apoyo a las comunidades, el compromiso de la escuela para liderar la armonización continua con un PEI adaptado al contexto y susceptible a la desescolarización, la tarea que deben cumplir el estudiantado para abordar con autonomía el proceso educativo y la importancia de que las familias sean preparadas por la misma escuela, para afrontar las necesidades que demanda la educación actual.
\end{abstract}

Palabras clave: pandemia, brecha digital, educación, análisis.

Abstract: This essay is carried out in order to publicize the difficulties that Colombian public education has faced and the inequity that has become visible with the arrival of the Covid-19 pandemic. For this, various difficulties are analyzed, such as the lack of a solid Institutional Educational Project, the absence of connectivity and the insufficiency of tools and technological knowledge of all the actors of the educational community, which have a background lack of knowledge in the management of digital tools and / or the scarcity of economic resources, as well as the absence of self-learning habits. Hence, it is necessary that all the actors involved in the educational process, with commitment, play the role that corresponds to them, in such a way that the curriculum is fully complied with, regardless of the circumstances that surround it. The conclusion establishes the responsibility that the State must assume in the allocation of resources for education and support to the communities, the commitment of the school to lead the continuous harmonization with an IEP adapted to the context and susceptible to unschooling, the a task that students must fulfill to autonomously approach the educational process and the importance of preparing families to face the needs of today's education.

Key words: pandemic, digital divide, education, analysis.

\footnotetext{
${ }^{1}$ Docente de Lenguaje, en Institución Educativa, Departamental Limoncitos, Cundinamarca, Colombia. Aspirante a Doctora en Ciencias de la Educación de la Universidad Arturo Prat de Chile, Magister en Educación, Especialista en gerencia educativa, Licenciada en Español y Literatura. Orcid https://orcid.org/0000-0002-8456-4342
}

Dirección electrónica: stellamonmah@gmail.com

Ensayo recibido: 5 de abril, 2021

Enviado a corrección: 21 de junio, 2021

Aprobado: 16 de agosto, 2021 


\section{Introducción}

La presencia del Covid 19, dio lugar a cambios drásticos en la vida de las personas, de manera particular, en la educación, por cuanto fue necesario estudiar y enseñar desde casa. Las dificultades más significativas están determinadas por la ineficacia del Proyecto Educativo Institucional, la ausencia de conectividad, la falta de herramientas y conocimientos tecnológicos originados por las carencias económicas, al igual que la inexistencia de hábitos de aprendizaje autónomo.

Es verdad que nadie estaba preparado para tal acontecimiento, por tanto, ello hizo que se generara incertidumbre en torno a las alternativas que deberían llevarse a cabo para garantizar la continuidad educativa y no afectar a las niñas, los niños y jóvenes que estaban en este momento cursando su educación preescolar, básica y media. Esta situación mostró la fragilidad de un sistema que, aunque ha tratado de robustecerse, aún no logra afianzar bases para anclarse como organismo sólido y darle el lugar que le corresponde en la sociedad. Como lo afirma Lorente (2018), para avanzar en mayores condiciones de equidad y movilidad social es imperativo que la educación sea parte indispensable en las organizaciones sociales, que sea uno de los ejes centrales de desarrollo social en las políticas de Estado.

El objetivo de este ensayo es visibilizar los inconvenientes que presenta la educación actual colombiana ante la llegada de la pandemia del Covid-19. De allí que es necesario señalar y analizar los retos del gobierno y de las comunidades educativas para afrontarla, especialmente aquellas de población vulnerable. Para ello se hace necesario hacer una indagación en diferentes documentos, medios de comunicación y a partir de la experiencia docente. Finalmente se busca dar un aporte que invite a la reflexión y quizá a la formulación de una política pública que lleve a solucionar estas dificultades en las que cada uno de los actores de la comunidad educativa asuman su responsabilidad.

Este documento está planteado en tres momentos: el primero hace referencia a la conceptualización de conectividad, Proyecto Educativo Institucional (PEI) y aprendizaje autónomo, en la segunda parte se habla de las dificultades de la educación colombiana en el marco de la Pandemia del Covid-19 y finalmente, se plantean alternativas que, si bien es cierto, no serán aplicables a esta dificultad, sí buscan preparar a la comunidad educativa para futuras situaciones similares. 


\section{La conectividad como recurso educativo indispensable}

Hablar de conectividad es referirse a un término usado en la actual globalización, que busca mantener en permanente e instantánea comunicación al mundo entero, en cualquier campo donde haya necesidad de relacionarse. Para acceder a este servicio es preciso contar con aparatos tecnológicos que permitan que los usuarios se interconecten. Esto se puede lograr mediante dispositivos tangibles que admitan a las personas adherirse a los mecanismos tecnológicos a través de las TIC (Tecnologías de la Información y la Comunicación) (García, Caldas, Dávila y Thoene, 2020). Restrepo y Gómez (2020) refieren que la conectividad digital representa independencia a través de herramientas digitales, que fortalecen y empoderan al ser humano. En este sentido, tener conectividad digital es gozar de un recurso indispensable y necesario que facilite el acceso a la información para que el sujeto desarrolle hábitos de aprendizaje autónomo y, a su vez, adquiera mayores conocimientos que posibiliten cumplir, no solo con las exigencias desde el campo educativo, sino desde otros ámbitos de la vida; de tal manera que el sujeto tenga la posibilidad de estar informado de diversas temáticas que hacen parte de la vanguardia del momento.

Desde esta perspectiva, es evidente que este servicio es limitado para los usuarios de la educación pública colombiana que, en su mayoría, no cuentan con recursos, ni físicos, ni económicos para acceder a el, lo cual hace que se creen brechas digitales. Según cifras del Banco Mundial, la conectividad a nivel universal es deficiente, como lo muestran las siguientes gráficas.

Figura 1

Porcentaje de población mundial sin acceso a conectividad, 2021

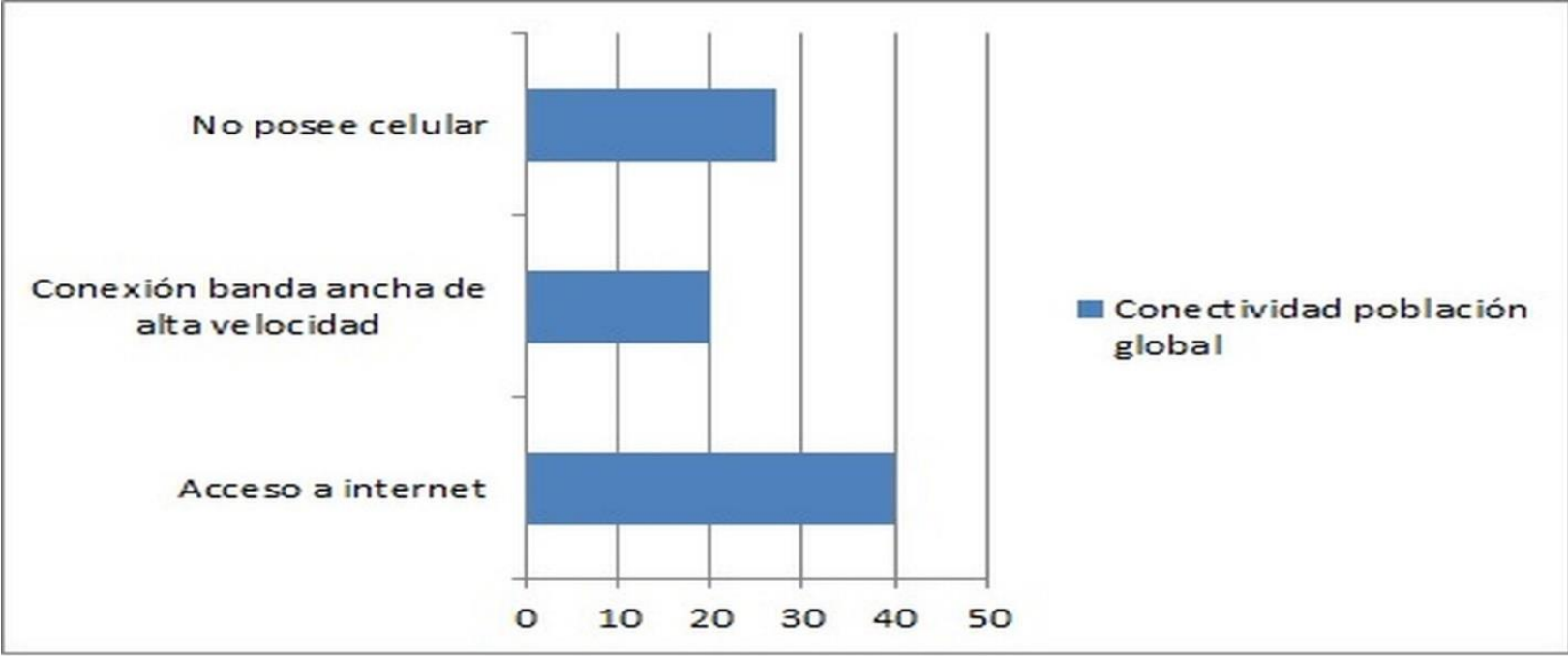

Fuente: Elaboración propia con base en documento SELA (2021), según cifras del Banco Mundial 
En la figura 1 se puede visualizar cómo a nivel mundial el acceso a internet es mínimo, pero mucho menor es la conexión a banda ancha de alta velocidad. Igualmente, se aprecia que casi un tercio de la población no cuenta con un equipo móvil, situación que refleja la inequidad en términos de acceso a equipos básicos para la conectividad digital. No contar con la posibilidad de acceder a herramientas para la interacción digital, limita procesos de comunicación efectiva y acceso a información disponible en la red. A su vez, dificulta crear redes de aprendizaje virtual que permitan fortalecer la formación estudiantil, profundizando, de esta manera, las brechas de inequidad en un contexto de pandemia.

Igualmente, conviene ver cómo los hogares de bajos ingresos son los que presentan mayores deficiencias de conectividad. Lo anterior, por cuanto estas familias, además de carecer de recursos económicos que les permita acceder a equipos tecnológicos, no pueden cubrir gastos de conectividad que, de cualquier manera, tampoco son subsidiados por el Estado.

Figura 2

Porcentaje de hogares con menores ingresos de la población mundial sin conectividad a internet ni acceso a teléfono móvil, 2021

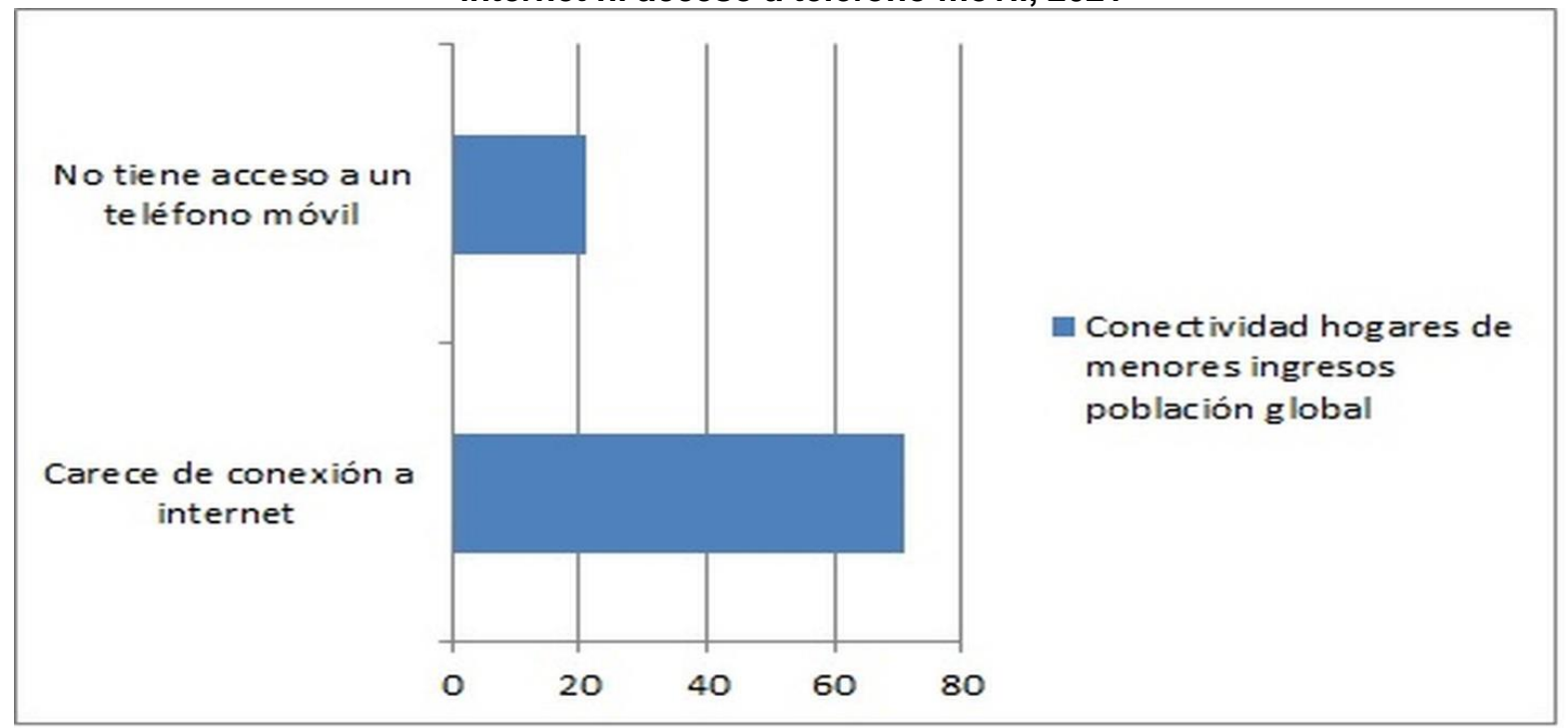

Fuente: Elaboración propia con base en documento SELA (2021), según cifras del Banco Mundial

La Figura 2 deja ver cómo a nivel mundial la gran mayoría de las familias con menores posibilidades económicas, carece de servicio de internet y un porcentaje significativo no cuenta con dispositivo celular. Lo anterior permite confirmar que la ausencia de recursos tecnológicos, como el espacio virtual y el teléfono móvil, representan una enorme brecha para el desarrollo efectivo de la comunicación de estas comunidades, en tanto no les consiente desarrollar de manera eficiente su proceso educativo. 
De otro lado, resulta conveniente analizar la situación de conectividad en América Latina y el Caribe, donde se compare la cobertura de conectividad de la zona rural y la zona urbana de la región, al igual que se revisen las posibilidades de conectividad existentes en las aulas.

Figura 3

Porcentaje de población y de escuelas de América Latina y el Caribe con falta de conectividad y herramientas de digitalización

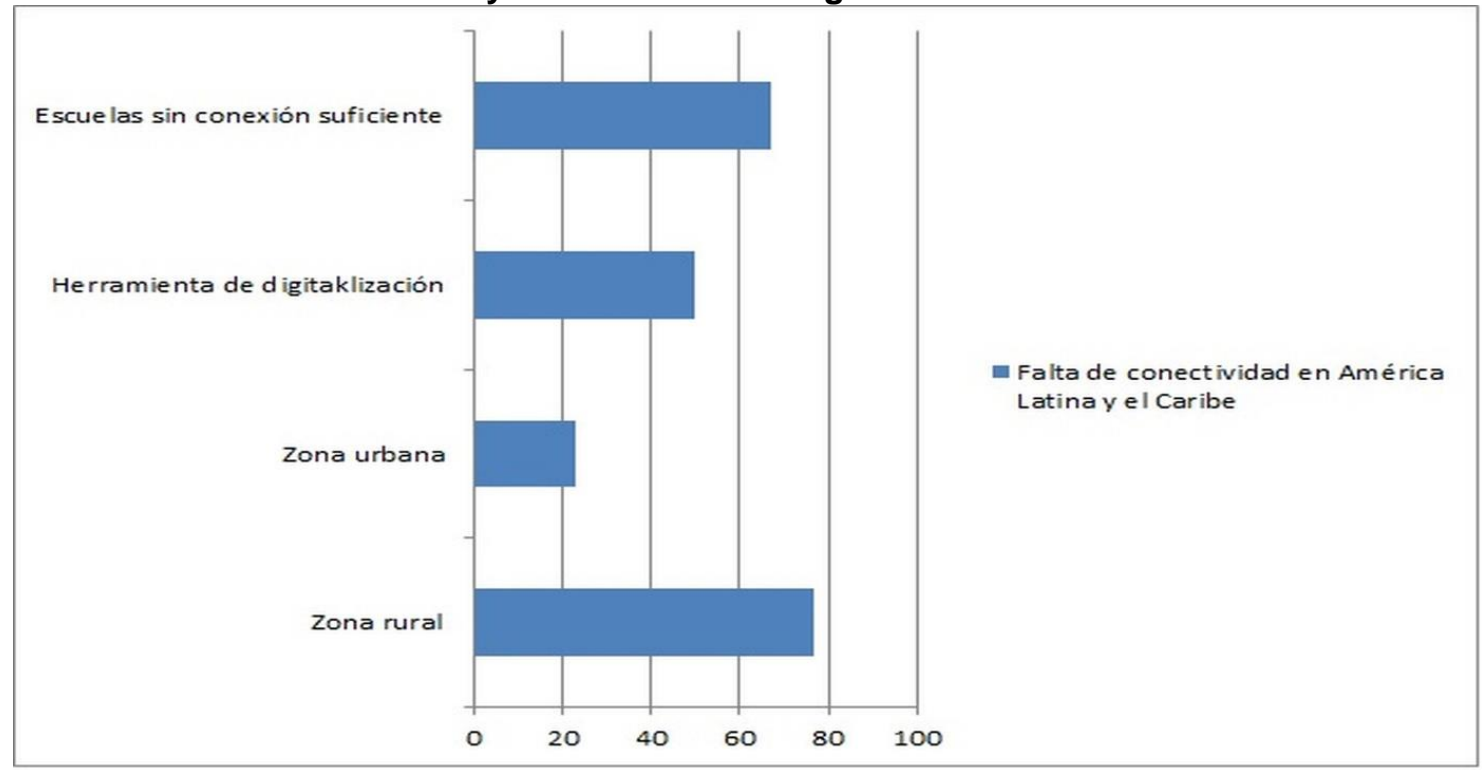

Fuente: Elaboración propia con base en documento SELA (2021), según cifras del Banco Mundial

En la Figura 3 se puede apreciar que la población de América Latina presenta mayores dificultades frente a la conectividad a nivel mundial. El sector rural es el que lleva la peor parte, por cuanto más de dos tercios carecen de conexión. La mitad de los habitantes no cuentan con herramientas tecnológicas tangibles, además que dos tercios de las instituciones no poseen adecuado enlace a internet. Los datos anteriores permiten visualizar que las zonas rurales y las escuelas son los sectores más afectados por la falta de conectividad suficiente. Es evidente que las escuelas del sector rural, que deberían tener mayor atención por su carácter de vulnerabilidad, son las que más dificultades de conexión a las plataformas de comunicación tienen. La anterior situación pone en notable desventaja a estas poblaciones que, de base, ya cuentan con menores oportunidades respecto a las comunidades urbanas, lo que acrecienta mucho más la brecha frente al derecho a una educación de calidad que les permita prepararse como parte activa en la construcción de país. 
Ahora, es conveniente analizar la realidad local de una comunidad rural colombiana en la que la autora interviene, de tal manera que se ponga en contexto una situación específica, que dé cuenta de lo que viven la escuela y las familias para estudiar y educar en pandemia.

En concordancia con lo anterior, resulta relevante fijar la mirada en los hogares de esta comunidad educativa, para determinar qué tipo de aparatos tecnológicos y de conectividad poseen.

Figura: 4

Aparatos tecnológicos y conectividad con que cuentan las familias de una Institución rural de Colombia

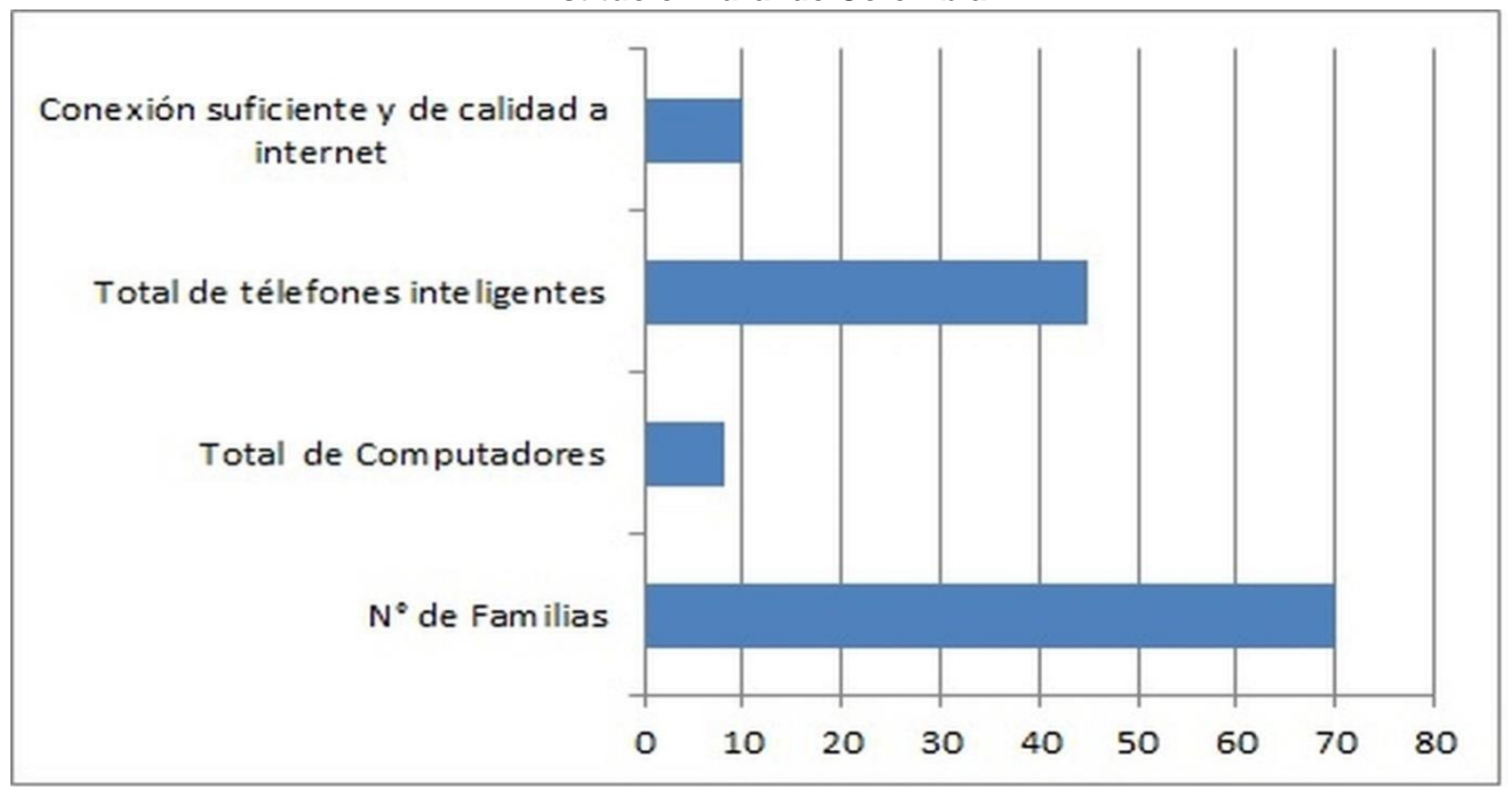

Fuente: Elaboración propia, 2021

En la Figura 4 se visibiliza como este establecimiento rural presenta un alto índice en la ausencia de herramientas tecnológicas, pero mayor aún en la conectividad. Se observa que esta institución se encuentra muy por debajo de los rangos a nivel mundial y latinoamericano, tan solo una mínima parte de la población escolar puede disponer de un equipo en red dentro de la escuela.

Por tanto, es evidente que las instituciones del sector rural colombiano enfrentan una limitante significativa en materia de acceso a una red digital, en la que se interconecten y accedan a información educativa o de cualquier otro tipo. Este panorama deja por sentado que la escuela se encuentra aislada de posibilidades reales en las que sus estudiantes puedan acceder a contenidos de la red, tampoco les permite intercambiar experiencias escolares. Igualmente, para sus maestros y maestras no es posible liderar procesos en los que las redes 
de información sean necesarias, lo que hace que esta población escolar quede rezagada frente a los aprendizajes que en otros entornos de carácter urbano sí son posibles, con lo que se genera una enorme desigualdad.

Figura 5

Porcentaje de población escolar con acceso a equipo digital y conectividad en una institución rural de Colombia.

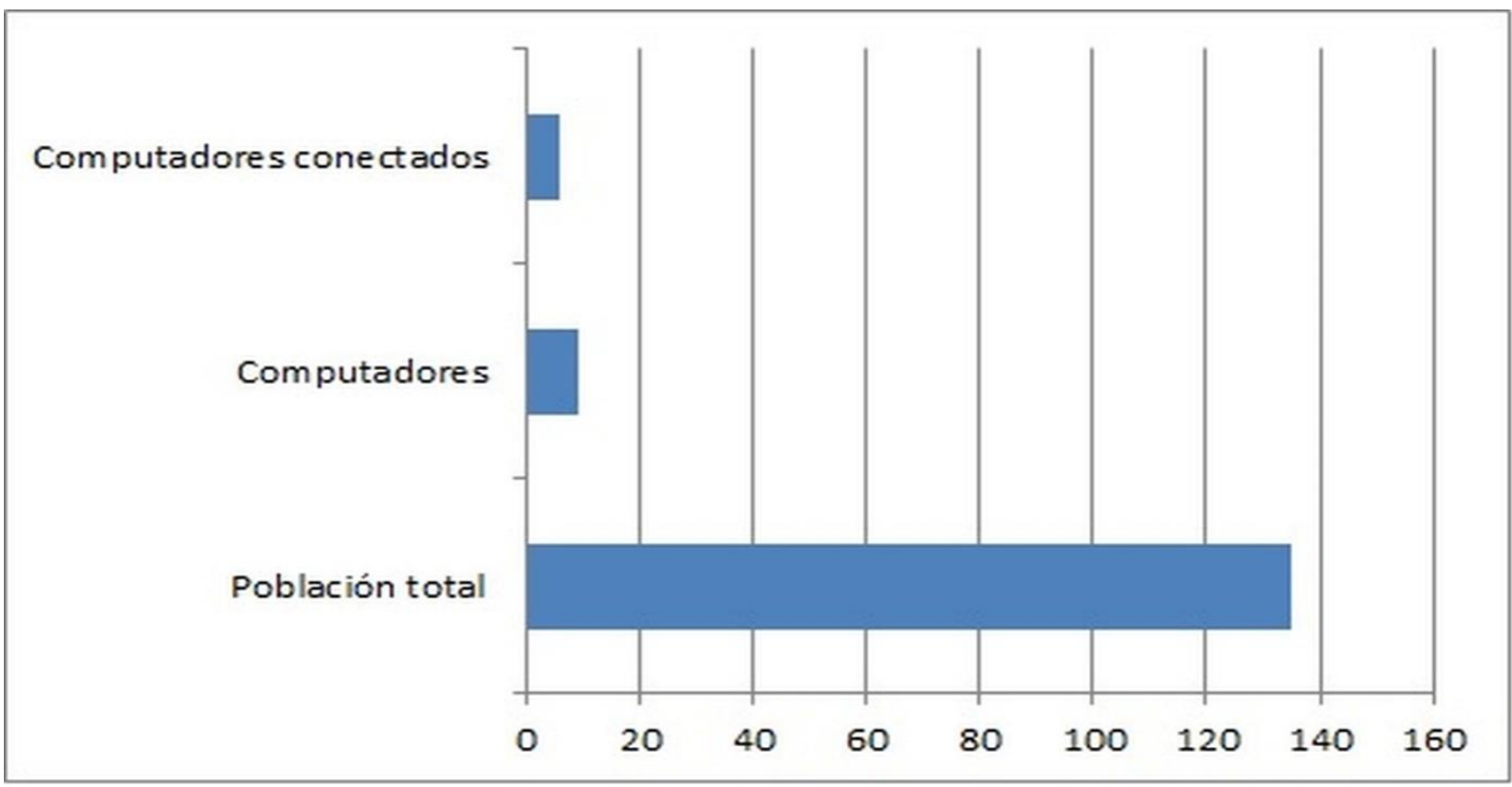

Fuente: Elaboracion propia, 2021.

La Figura 5 permite determinar que, aunque la conectividad es bastante deficiente, lo es más la de la institución educativa. Se observa que tan solo una sexta parte de los hogares cuentan con un computador, un poco más de la mitad de familias tiene un teléfono inteligente, pero solo una décima parte puede disfrutar de conexión suficiente y de buena calidad.

Ante un panorama como el presentado anteriormente no es posible pensar que el avance del proceso académico, en medio de la pandemia, resulte efectivo. Son muchas las limitantes que impiden el desarrollo de las actividades pedagógicas en donde la conectividad es solo una de ellas; sin embargo, es la de mayor trascendencia por cuanto se constituye en el recurso de comunicación para las comunidades. En este contexto aflora la frustración de unos y otros; el cuerpo docente por no poder hacer un acompañamiento pertinente, el estudiantado porque no tiene ningún aliciente que les motive a seguir con su educación desde casa y los padres y las madres porque no cuentan con los recursos económicos suficientes para brindarle a sus hijas e hijos las herramientas tangibles y la conectividad que requieren. 
Como se puede apreciar, el panorama desolador devela la gran fractura educativa y la inequidad existentes en esta institución rural. A pesar de que han transcurrido varios meses y que a nivel estatal se han planteado diversas soluciones para mejorar la conectividad, esto no se ha superado, la comunidad sigue como si el tiempo no transcurriera, no ha habido cambio, ni progreso de acceso a las tecnologías de la información que permita transformar el entorno educativo en una comunidad académica activa.

\subsection{Proyecto educativo institucional y aprendizaje autónomo}

La hoja de ruta por excelencia de cualquier institución educativa Colombiana es el PEI (Proyecto Educativo Institucional), el cual, de acuerdo con la Ley 115 de 1994, en su artículo 73, establece que toda institución educativa debe elaborar y poner en práctica su PEI, para tener claro los derroteros a seguir en cuanto a los "principios y fines del establecimiento, los recursos docentes y didácticos disponibles y necesarios, la estrategia pedagógica, el reglamento para profesores y estudiantes y el sistema de gestión" (Colombia, Ministerio de Educación Nacional, 1994), esto por supuesto ciñéndose a la Ley General de la Educación.

En este sentido, las diferentes instituciones educativas tienen la libertad de construir su propio PEI, adaptándolo a las necesidades y al contexto. Con todo ello se debería prever cualquier eventualidad y estar preparados para que acontecimientos sorpresivos como la pandemia del Covid-19, no sorprendan a las comunidades escolares, las que a pesar de las situaciones complejas deben continuar con el desarrollo de las actividades académicas.

Dicha carta de navegación de cada institución debe estar legitimada desde la autonomía que le brindan las políticas educativas, además, se deben evidenciar las fortalezas de todo plantel educativo. Tonucci (2020), afirma que las instituciones de este siglo han de ser sitios donde los sujetos puedan mostrarse y desenvolverse, que hallen sus objetivos puesto que deben ser lugares abiertos a diversas posibilidades. Desde esta perspectiva, un proyecto educativo sólido debe garantizar que aún sin la presencia del personal docente o de la no asistencia a la escuela, el proceso continúe, siempre y cuando se cuente con las herramientas necesarias, proveídas por el Estado, para tal fin.

Ahora, ser autónomo significa valerse de su propio criterio para realizar cualquier actividad o tomar decisiones, sin tener que recurrir al otro para que le indique lo que debe hacer. Bajo esta perspectiva se entiende como aprendizaje autónomo el tener discernimiento en el actuar, reflexionar y analizar, con seguridad y disposición, aplicando juicios asertivos de manera libre y con propiedad, valiéndose de conocimientos previos. Este es un concepto 
actual, que va de la mano con procesos de enseñanza a distancia y tecnologías de la información, que permiten al sujeto adaptar los tiempos para cumplir los objetivos (Escobedo, Pimentel y Cruz, 2020).

En este sentido, el aprendizaje autónomo se adelanta promoviendo en el sujeto la participación activa, mediada por autoaprendizaje, que involucra destrezas que permiten aprender a aprender (Chica, 2010). Ese aprender, es saber qué se debe hacer y en qué tiempo y momento se ejecutan las tareas a entregar, es concebirse como autorregulador de su proceso.

Así, para potenciar hábitos de aprendizaje autónomo en las niñas y los niños, es conveniente que desde el PEI se propongan estrategias, que a mediano y largo plazo conduzcan al desarrollo de actividades académicas sin la presencia docente. El asignar actividades sencillas que a diario debe cumplir el estudiantado, como parte de su rutina escolar, es el inicio del camino hacia la autonomía, igualmente el plantear la clase a manera de taller, donde se deban desarrollar tareas bajo la supervisión docente, pero con el concurso personal del estudiantado, va consolidando el propósito en cuestión. Otra estrategia, no menos efectiva, puede resultar el aprendizaje cooperativo, en el que las personas aprendices tienen el compromiso propio y el de sus pares, para lograr objetivos colectivos (Martínez y Sánchez, 2020) que buscan fortalecer responsabilidades individuales tendientes a la consecución de metas comunes.

Peinado (2020) refiere, que el aprendizaje autónomo se da cuando la persona estudiante plantea posibilidades de solución, asumiendo y cumpliendo sus responsabilidades, para ello se deben direccionar adecuadamente las metodologías para que sean utilizadas de manera eficaz, esto requiere que el estudiantado se tenga confianza y crea en sus potencialidades. En suma, es el proceso que faculta a los seres humanos para conseguir habilidades de pensamiento, metacognición, y destrezas de tendencia global (Solorzano, 2017).

\section{Argumentos para la discusión de las dificultades de la educación colombiana en el marco de la pandemia del Covid-19. La educación en Colombia retos y realidades}

Momentos vividos como el de la actual emergencia sanitaria, a nivel mundial, llevan a reflexionar de qué manera el "sistema educativo, como producto de un sistema social, se afecta por los cambios sociales provocados por factores externos, que necesariamente se reflejan en los factores internos del proceso educativo". (Solovieva y Quintanar, 2020, p. 6). 
Desde esta mirada, el siguiente apartado señala algunas dificultades que han tenido que sortear las comunidades educativas, en el desarrollo de la enseñanza aprendizaje en época de pandemia del Covid-19.

Vale la pena citar cómo la educación colombiana ha presentado grandes tropiezos a lo largo de su historia, también es evidente que la lucha de reivindicación de la misma, ha sido incansable; "la preocupación por la calidad de la educación ha sido una constante desde finales del siglo XIX, a partir de los procesos de institucionalización de la educación pública y de masa" (Chacón, 2019, p. 1). Personal docente, estudioso de los problemas educativos, padres de familia y el mismo Estado, entre otros, han dedicado significativos esfuerzos a mejorar la eficacia educativa, quedándose todos ellos apenas en leves cambios que resultan insuficientes para lograr el nivel educativo con que deberían contar el estudiantado. Y de pronto aparece un monstruo invisible y escurridizo que se expande por el mundo entero para causar pavor, obligando al aislamiento en aras de preservar la vida. Entonces, como si la escuela no tuviera problemas para ser atendidos, la enseñanza se debe volver global y fluctuar de hogar a hogar, desde la casa de las familias, hasta la de sus docentes, pasando por la de las personas directivas, así que las contrariedades se multiplican.

Es evidente que la educación se vio desequilibrada con la llegada de la crisis sanitaria, puesto que la escuela no estaba preparada para asumir tal acontecimiento. Cuando el gobierno central por obvias razones decretó "el aislamiento social preventivo" (Gobierno Nacional de Colombia, Decreto 457, 22 de marzo de 2020) en el que envió a docentes y estudiantes a trabajar en casa sin un "lineamiento general" (De Zubiría, 2020) y sin políticas claras que permitieran realizar el trabajo a la distancia, se hubiera esperado que las instituciones educativas estuvieran capacitadas para continuar con la educación desde cualquier lugar, que no fuera el contexto de la planta física llamada "institución educativa", pero no fue así, las dificultades que se percibieron fueron variadas, complejas y hasta inhumanas.

La principal dificultad que se debió sortear está relacionada con la comunicación entre los miembros de la comunidad educativa, especialmente entre maestras y maestros con sus estudiantes. No obstante, la virtualidad tuvo que ser implementada sin contar con la suficiente preparación para su uso o incluso con la carencia de este recurso (Cajiao, 2020).

La falta de herramientas tecnológicas y de conectividad se hizo sentir y lo hace notar la Unión Internacional de Telecomunicaciones (UIT) que muestra que en el caso de “...la penetración de Internet móvil, Colombia se encuentra en la posición 19 entre 27 países de América, con 48,8 conexiones activas por cada 100 habitantes en 2017", (Ministerio de 
Tecnologías de la Información y las Comunicación de Colombia [MIN TIC], 2019), lo cual no representa ni siquiera la mitad de la población. A eso se le suma que el mayor porcentaje de conectividad se halla en la zona urbana, el sector rural lleva la peor parte en el rezago tecnológico, lo que repercute notablemente en el desarrollo de la educación básica y Media del sector público, donde acude la población más vulnerable del territorio.

La preocupación por la insuficiencia de herramientas tecnológicas es general, en toda la comunidad educativa y especialmente en docentes que están conscientes de las falencias de este sistema y la falta de conectividad del estudiantado.

Es insólito que en pleno siglo XXI, la gran mayoría de comunidades no pueda contar con conectividad por diversos factores. Hay quienes tienen su móvil con redes sociales y aunque realicen una pequeña recarga no disfrutan de señal y se deben desplazar a una hora o más de camino para enviar un trabajo o hacer una consulta. Padres, madres y/o acudientes que no cuentan con celular de alta tecnología que les permita conectarse a internet, otros que no tienen fluido eléctrico, o a quienes se les daña la electricidad y se ven obligados a esperar cierto tiempo para que se les restablezca el servicio, o simplemente aquellos que no tienen un dispositivo de ningún tipo.

En el mismo sentido, existen varias zonas que se creería apartadas, pero no lo están tanto, o tal vez sí, alejadas de la ayuda del gobierno, puesto que distando a dos horas de la capital no han podido acceder a las clases, ni siquiera por TV o radio. Estos medios son privilegio de pocos, por diversas razones, de un lado porque en algunas ocasiones las familias, aunque suene increíble, no cuentan con TV o radio; o si lo tienen, no se ha brindado la suficiente información para adherirse a las clases, o la señal no llega, "no cogen todas las emisoras", o se les termina la batería. Aunque parezca curioso, es la cruda realidad que viven día a día muchas familias colombianas rezagadas en el olvido y sin oportunidades de salir adelante, en un país tan desigual que pareciera sacado de un cuento de terror.

Las estrategias adoptadas para que el estudiantado siguiera "yendo a la escuela desde casa", fueron diversas, pero los recursos para acceder a ellas también limitados. De Zubiría (2020), afirmó que, en Colombia, únicamente "el $4 \%$ de los municipios tiene buena conectividad, el 63\% de los bachilleres del 2018 no tenía acceso a Internet desde sus hogares y, en las zonas rurales, tan solo el $9 \%$ de los jóvenes disponen de computador". Ante este panorama se puede ver que los niveles de ineficiencia en conectividad son bastante altos, lo que repercutió en aumentar las desigualdades e hizo que la brecha entre unos y otros se hiciera más visible. 
No cabe duda que estas abruptas transformaciones que se están viviendo, no le cayeron bien a nadie, pues fue tan sorpresivo e intempestivo el cambio, que sacó a todos los entes de su zona de confort y los puso en una situación de inmediatez que no tenían calculada. Y si a todo esto se le suma el choque emocional por el que se tiene que atravesar en un escenario como este, se agravan las condiciones de vulnerabilidad, para las clases menos favorecida (Johnson, Saletti y Tumas, 2020), que al no tener un horizonte claro de los derroteros a seguir para afrontar las diversas situaciones de índole psicosocial, económico y educativo, están expuestos a la deserción escolar, al maltrato, tal como lo señala la Unesco - Cepal, en el periódico El País, (2020) al decir que "además de interrumpir las trayectorias educativas, el cierre de las escuelas afecta la alimentación y la nutrición de la población estudiantil, especialmente en los sectores más vulnerables" (p.3).

Las dificultades de orden tecnológico y de conectividad, se originan en la precariedad de recursos económicos que giran cada año a las instituciones para mantener un servicio educativo digno y de calidad. Dicha asignación no es suficiente para que accedan a herramientas eficaces que beneficie al estudiantado, teniendo en cuenta que el Sistema General de Participación (SGP), establece que estos recursos deben ser destinados a “: i) población atendida; ii) población por atender en condiciones de eficiencia y iii) equidad" (Ley 1176, 2007).

En concordancia con lo anterior, obsérvese cómo una institución pública con 663 estudiantes, en el año 2020 le asignaban un presupuesto promedio de $\$ 56.000 .000$ millones de pesos, si se hace la división, le corresponderían \$84.464 pesos a cada estudiante, para utilizarlos en infraestructura, pago de servicios, material didáctico, insumos y papelería, entre otros. Es así que se aprecia que esta inapropiada suma, no alcanza para los diversos gastos que tiene una institución educativa para cumplir con los proyectos en beneficio de la formación estudiantil. Frente a la situación actual, se corre el riesgo de que “... esta pandemia deje como saldo negativo un aumento en las grandes desigualdades de nuestra sociedad, ya que los mayores costos son afrontados por los alumnos de las escuelas localizadas en las áreas donde habitan los más humildes" (Guadagni, 2020, p.4).

Además de la escasa asignación presupuestal a las instituciones educativas, debe tenerse en cuenta también las brechas de ingresos e inestabilidad laboral entre el sector rural y urbano. Un campesino cuyo salario surge de la venta de sus cosechas en una zona desfavorecida por las políticas agropecuarias y que depende del jornal diario, sin acceso a la propiedad de la tierra, difícilmente cuenta con posibilidades reales para apoyar a sus hijos en 
la adquisición de medios tecnológicos y conectividad para acercarlos un poco a la educación asistida por las TIC.

En concordancia con lo dicho hasta el momento, es necesario, que desde el gobierno central se prioricen y aumenten los recursos para la educación, teniendo en cuenta las necesidades individuales de cada centro educativo, buscando favorecer de manera especial a los más lejanos y apartados.

Igualmente, es necesario señalar que los textos existentes son insuficientes o están desactualizados. Al ser escasos, no es posible asignar uno a cada estudiante para que continúen su proceso en casa con la orientación del docente, la que solo es posible atender por una llamada o por WhatsApp. Al estar desactualizados no permiten que se desarrollen las competencias básicas necesarias para la educación actual, entre las que se cuentan las científicas, las ciudadanas, las comunicativas y las matemáticas" (MEN, 2016). Aunado a ello el proceder del estudiantado, la falta de compromiso, la ineficiencia de herramientas tecnológicas y la ineficacia para utilizarlas, hace que la situación sea más compleja y difícil de solucionar.

Así que la paralela mezcla de ambientes y dificultades ha suscitado una verdadera combinación de faenas e inquietudes que no se habían visto con anterioridad, tomando desprevenidas a las instituciones, al colectivo de sujetos intervinientes en el proceso educativo y a toda la comunidad (Sánchez, Martínez, Torres, De Agüero, Hernández, Benavides... y Jaimes, 2020).

No se pueden desconocer los esfuerzos que hace el gobierno para robustecer los centros educativos y suscitar una conveniente gestión (MEN, 2016). El estado cuenta con contratación de expertos para brindar ciertas capacitaciones, tanto a personal directivo, como a docentes; entre ellos está el programa de becas para especializaciones, maestrías y doctorados; el problema radica en que no es para todas las personas, solo algunas pocas tienen el privilegio de suscribirse a estos.

Otro recurso que brinda el MEN a la comunidad en general, es la página educativa Colombia Aprende, donde se encuentran contenidos pedagógicos interesantes para hacer uso de ellos en el momento que se requiera. La falla está en que no se ha brindado la información necesaria para que el profesorado y demás integrantes de la comunidad accedan a estos recursos, además que ni la institución, ni los hogares cuentan con conectividad para tal fin. 
Es verdad que nadie estaba preparado para vivir una pandemia como la actual, pero sí se han presentado sucesos que permitieron analizar y prever realidades que proporcionaran un plan B para asumir la educación fuera del establecimiento educativo. Cabe mencionar solo algunos de los eventos donde el estudiantado se desescolariza, bien sea por enfermedad, por maternidad, por incapacidad docente, o por cualquier otra situación en la que no pueden asistir por varios días o hasta meses, lo que no debería entorpecer el desarrollo pedagógico, pues un banco de guías por grados y materias que estuvieran adaptados a los contenidos pedagógicos, hubiera sido una solución pensada previamente desde el PEI.

La cuarentena o aislamiento social preventivo obligatorio, tal vez fue el reto más grande porque todo el país debió confinarse a pesar de las dificultades y consecuencias que eso acarreó. Con gran preocupación el presidente Iván Duque Márquez, a través de los medios de comunicación masiva declaró el confinamiento preventivo obligatorio, y en su momento, respecto a la escuela manifestó textualmente: "cada Institución Educativa y sus docentes determinarán las estrategias, recursos y guías para el plan de trabajo académico en casa, de acuerdo con las características de las familias, las edades de los estudiantes, los recursos disponibles...". MEN (2020a, p.1), donde las opciones no eran tantas para la gran mayoría del territorio, en el que se presentan más carencias que excesos y que dejaron en evidencia las grandes brechas existentes. $Y$ es que un sistema educativo incipiente e ineficaz se traduce en miseria e inequidad (Vijil y Castillo, 2020).

Otro aspecto que se debió afrontar fue la modificación del calendario escolar, en el que de pronto enviaron de manera abrupta a estudiantes, personal directivo y docentes a "vacaciones anticipadas" (MEN, 2020b, Circular № 020), así que no se pudo tener en cuenta lo planeado desde el PEI. Lo anterior, como solución a la falta de planes de contingencia del Estado para atender emergencias y que sin duda perjudica tanto a estudiantes, como a docentes. Los niños y las niñas siempre esperan esta etapa para el disfrute del tiempo libre, visitar y compartir con sus familias, departir con sus compañeros en otros espacios que no sean los educativos, dejar la rutina de levantarse temprano; y de pronto los envían a casa a confinarse sin que los más pequeños entiendan lo que está ocurriendo. Es paradójico que en este momento deseen volver a las aulas, que ya no quieran continuar con la educación en casa, quieren ir a su institución a compartir con sus amigos, extrañan a su docente, porque no es lo mismo estudiar en la casa que en el colegio, es más "chévere" ir al colegio, según lo afirmaron algunos niños entrevistados en alguna cadena televisiva. $Y$ es que es así, en las 
instituciones socializan, comparten con sus pares, juegan, aprenden, se fortalecen, asumen responsabilidades y confianza en sí mismos.

Docentes, niñas, niños, jóvenes y familiares se vieron ante un gran desafío: adecuar los modelos tradicionales, al contexto actual, y lograr el mayor beneficio de esta situación (MEN, 2020). El proceso ha sido complejo, pues los cambios de paradigmas siempre traen con consigo dudas, incertidumbre; sin embargo, es necesario señalar que estas consideraciones no son nuevas, cada día el cuerpo docente debe navegar en un mar de situaciones que han de ser resueltas en su contexto educativo, en el que hacen uso de su potencial creativo para no dejar derrumbar su institución, sus ideales y objetivos. Es así como a marcha forzada tuvieron que adoptar diferentes métodos para llegarle al estudiantado, entre ellos: guías, talleres, llamadas, mensajes de WhatsApp, entre otros.

El reto ha sido educar al estudiantado para que maneje su tiempo, realice y entregue actividades establecidas en la nueva modalidad, que no se podría llamar, virtual porque no tienen este servicio, tal vez remota o a distancia, la cual no estaba contemplada en ningún PEI de la educación regular. No ha sido fácil para el estudiantado trabajar de manera independiente, sin el acompañamiento permanente y presencial del cuerpo docente, como tampoco ha sido efectivo el apoyo de las familias. Entre el estudiantado está el que se preocupa por desarrollar su trabajo, el que hay que llamar a menudo para preguntarles sobre las dudas y resolverlas, y al que se le debe repetir en varias ocasiones lo mismo para que entienda, incluso el que no se interesa por el trabajo y hay que motivarles a diario.

En este orden de ideas y para subsanar el inconveniente de la conectividad, el profesorado ha tenido que planear, diseñar y entregar guías, acción que para algunos no ha sido muy fácil, teniendo en cuenta que muchos no son diestros en el manejo del computador o no cuentan con esta herramienta en casa. De modo que ha sido necesario adquirir equipos de cómputo, buscar ayuda de terceros y aprender bajo la necesidad, lo que evidencia una vez más el desafío tecnológico del momento. Es conveniente reflexionar acerca de la necesidad de que el cuerpo docente incursione en el uso de metodologías activas y esté a la vanguardia en el uso de las TIC, para lograr el cambio en la manera de enseñar en la escuela.

En el mismo sentido, es conveniente señalar la afectación de docentes, quienes están en constante estrés al trabajar con un promedio de 250 estudiantes al día durante cada periodo académico. Así que, el alto volumen de trabajo ha requerido que se extiendan las jornadas laborales fuera de los horarios establecidos, convirtiendo el hogar en una escuela permanente que les impide gozar del descanso necesario, reponer energías y cambiar de rutina en aras 
de mantener su salud física y mental, como un derecho consagrado en la carta magna (Constitución Política de Colombia, 1991), en el que afirma que los trabajadores, independientemente de su condición, pueden gozar de su descanso y dedicar el tiempo a lo que consideren.

Todo lo anterior debe redundar en un solo objetivo que es el de sacar adelante esta situación, cuidando de que la población estudiantil continúe en el sistema y no se produzca deserción escolar, lo que aumentaría la precariedad de condiciones y la inequidad que existe en este momento. Es claro que para el profesorado es muy importante que el estudiantado menos favorecido se mantenga en el sistema escolar a pesar de todas las complejidades que se estén dando. Para ello es necesario un gran compromiso por parte de las personas educadoras, donde se estrechen lazos de solidaridad para actuar en bien de la niñez y las juventudes y se propenda en todo sentido por el bienestar de las (os) educandos, puesto que "solo los buenos maestros podrán salvar la escuela" (Tonucci, 2020, p. 1).

Cabe señalar que la Constitución Política de Colombia (1991), en su Artículo 67 promulga que la educación es un derecho y un servicio público en el que el sujeto accede al "conocimiento, a la ciencia, a la técnica, y a los demás bienes y valores de la cultura". Desde este contexto, la igualdad de condiciones debe primar en la búsqueda de una educación equitativa que propendan por la formación de niñas, niños y jóvenes sin ningún tipo de distinción, entendiendo que este derecho establecido desde la carta magna ha de ser inalienable y por ello, tanto las políticas públicas, como los proyectos pedagógicos que la regulan deben estar encaminados a sortear las diversas dificultades y circunstancias que se presenten en el devenir del día a día.

Se hace necesario replantear la escuela de tal manera que esta logre una educación de calidad (Barroso, Ardini y Corzo, 2020), una educación inclusiva. Esta educación inclusiva se consigue si se les da a las familias el lugar que les corresponde para que se conciban parte viva de la colectividad pedagógica, donde encuentren ambientes agradables y se sientan indispensables en los procesos inclusivos (Simón y Barrios, 2019).

Ahora bien, los recursos pedagógicos deben servir de apoyo para educar también a las familias, las que a su vez serán el sostén de la escuela. Es momento de que los padres y acudientes comprendan que la formación es un proceso compartido entre institución y familia puesto que ha sido en los últimos años encargado a los docentes (De León, 2011).

En este sentido y retomando las palabras del MEN (2020), la ejecución del trabajo académico autónomo en casa no es sencillo, constituye un desafío para docentes, estudiantes 
y familias. Es indispensable trabajar en equipo y de manera conjunta para lograr los objetivos, donde la escuela y la familia sean los principales agentes garantes de que el proceso educativo se lleve a cabo teniendo en cuenta el contexto y las circunstancias de cada comunidad educativa, lo que redunde en mejores posibilidades estudiantiles. Teniendo en cuenta que, mediante la instrucción directa o mediante la orientación proporcionada en el aprendizaje autodirigido, en modos sincrónicos o asincrónicos, el maestro sigue siendo esencial para dirigir el aprendizaje de los estudiantes (Organización para la Cooperación y el Desarrollo Económicos (OCDE, 2020).

Se hace necesario ratificar que entre más pronto se implemente una solución apropiada a la enseñanza no presencial mediante las Tics, mejorarán las posibilidades educativas a nivel general (UNESCO, 2020), con disposición de conexión a internet que estén presentes en todo el territorio para que la gran mayoría de las personas haga uso de ellas (Ministerio de Tecnologías de la Información y las Comunicación de Colombia [MINTIC], 2019).

Frente a estas dificultades que se afrontan a diario en el quehacer pedagógico, el conjunto de los diferentes actores inmersos en la problemática abordada, debe revisar su actuar encaminado a dar soluciones a corto, mediano y largo plazo. Acciones dirigidas a que el proceso de enseñanza aprendizaje posibilite que la actividad educativa continúe, independientemente de los espacios o circunstancias. Es entender que la escuela debe seguir vigente, a pesar de las situaciones y ambientes que la rodeen, pues solo variarían las diversas condiciones de acuerdo al contexto situacional, sin que las vicisitudes interfieran en la consecución de objetivos y el alcance de las metas.

Corresponde a la institución educativa contar con un $\mathrm{PEI}$ (Proyecto Educativo Institucional) adaptado a la realidad de cada centro educativo, que propenda por el bienestar y el futuro del estudiantado, y que el profesorado y personal directivo docente, se encarguen de hacerlo funcional. Es necesario adecuar herramientas pedagógicas que desde el plan de estudios y los planes de área permitan contar con derroteros a seguir en cualquier momento del año escolar y que esto no deje de lado ninguna de las áreas del conocimiento, ni de los proyectos transversales y de aula que se hayan propuesto con anterioridad. Son tiempos difíciles, donde todos los estudiosos de la educación deben unir conocimientos que propendan por el bienestar de la niñez y de la juventud en formación.

En cuanto a los estudiantes y sus familias, se hace necesario que asuman el rol que les corresponde. Los primeros empoderándose de su proceso de formación que implica abordar los conceptos y apropiarse de ellos, al igual que trabajar procedimientos y reflexionar saberes 
de manera consciente y responsable. A los segundos, velar y apoyar a sus hijas e hijos o acudidos para que cumplan integralmente con su papel de aprendientes del proceso educativo, de tal manera que se conviertan en transformadores de sociedad.

Lo expuesto aquí es imposible que se haga efectivo, si el Estado por su parte no es garante de una educación de calidad que implica la presencia de docentes y recursos suficientes y eficaces para el sostenimiento de la escuela. Una verdadera educación en la era de las comunicaciones, debe ser concebida desde una sociedad de la conectividad donde todos los actores de la comunidad educativa estén inmersos en ella, para lo que resulta pertinente pensar en la aldea global, tal como lo expusieron Mcluhan y Powers (1989), que hace evidente la manera como las tecnologías de la información se toman al mundo. Ante ello, Flores (2020) afirma que "el advenimiento de las tecnologías digitales generó cambios tan profundos que impactaron no solo en los procesos de producción, sino en la manera en la que las personas viven su cotidianidad" (p.1), cotidianidad que ya no puede estar apartada de las tecnologías de la información.

Por tanto, el Estado debe promover una política pública en la que las comunidades cuenten con acceso a redes de la información para realizar procesos completos. Además, es necesario que las políticas no se queden en el papel, sino que se hagan realidad extendiendo redes de conexión, sin costos para la población vulnerable, en todos los rincones del país. El impulso de conexión digital con el que el orden gubernamental provea a las comunidades con mayores necesidades, redundará en aprendizaje servicio y estimulará el interés colectivo por la adquisición de equipos tecnológicos (Comisión Económica para América Latina y el Caribe [CEPAL], 2020).

\section{Conclusiones}

Lo presentado anteriormente permite reflexionar acerca de lo imperioso que resulta una actitud concordante de las personas protagonistas con las necesidades de la comunidad, de lo contrario, la escuela deja de ser el ente vivo, jalonador de procesos sociales. La crisis sanitaria demostró la inmensa brecha que subyace a la realidad de la educación y de sus integrantes, en la que se hace preponderante la evaluación y reconsideración en general de todo el proceso educativo, en donde las funciones de cada uno de los actores sean revisadas, analizadas y reflexionadas.

Los inconvenientes del sistema educativo se dan principalmente por la carencia de recursos económicos, didácticos, físicos y humanos que se asignan para dicho fin. En 
concordancia con esto se hace necesario que se tomen iniciativas en las que, a través de las políticas públicas, la educación se haga más competitiva e igualitaria, que permita transformaciones estratégicas para innovar la escuela de manera clara, armónica y trascendental, hacia una educación inclusiva, masificada, que no desconozca las individualidades.

Desde el anterior panorama, se visualiza al profesorado como la columna vertebral de la enseñanza. Así que vale la pena darle un vistazo analítico a las falencias que existen en la academia, con el propósito de fortalecer las fragilidades e impulsar el conocimiento con mecanismos de formación. Se busca abordar la educación desde una mirada en la que las reestructuraciones que se vienen dando, sean efectivas en bien de la calidad de la misma, con profesionales transformadores e instituciones renovadoras, que combinen el aprendizaje creativo con las potencialidades perennes en el espacio social llamado escuela, a la vez que se orquestan verdaderos cambios donde los mitos se conviertan en realidades que permeen la comunidad educativa en general.

A la familia, el núcleo de la sociedad, se le deben brindar garantías que promuevan el desarrollo de una sociedad justa y equitativa, en la que los privilegios no sean individuales y la dignidad humana sea parte del colectivo, la sociedad se armonice, la educación se vuelva accesible y oportuna, el conocimiento sea inagotable y el renacer del individuo sea una carta abierta a la integralidad de condiciones, dignas, igualitarias, que sean el común denominador para la colectividad.

Por último, la compensación para las diversas dificultades que se vienen dando es promover y aplicar de manera sistemática políticas públicas que brinden herramientas necesarias en busca de equidad y sostenibilidad, en las que la toma de decisiones sea ecuánime para que el sistema educativo se fortalezca y por ende mejore la calidad educativa, sin que sea un privilegio para unos pocos, por el contrario que se dé para el grueso de la sociedad. 


\section{Referencias}

Barroso, María Belén., Ardini, Claudia. y Corzo, Leonardo. (2020). Herramientas digitales de comunicación en contexto COVID 19. El impacto en la relación estudiantes-instituciones educativas en Argentina. Revista ComHumanitas, 11(2), 98-122. doi: https://doi.org/10.31207/rch.v11i2.251

Cajiao, Francisco. (2020, 17 de abril). Que todos pasen el año: ¿la alternativa educativa ante la pandemia? El Tiempo. Recuperado de https://www.eltiempo.com/vida/educacion/coronavirus-colombia-hoy-alternativaeducativa-ante-pandemia-ultimas-noticias-485208

Comisión económica para América Latina y el Caribe (CEPAL). (2020). Universalizar el acceso a las tecnologías digitales para enfrentar los efectos del COVID 19. Informe especial COVID $19 . \quad$ Recuperado de https://repositorio.cepal.org/bitstream/handle/11362/45938/S2000550 es.pdf?sequence $=4 \&$ isAllowed $=y$

Chacón, Luis Francisco. (2019). Calidad Educativa: Una mirada a la escuela y al maestro en Colombia. Educación y Ciudad, (36), 35-49. Recuperado de https://revistas.idep.edu.co/index.php/educacion-y-ciudad/article/view/2120/1945

Chica, Francisco. (2010). Factores de la enseñanza que favorecen el aprendizaje autónomo en torno a las actividades de aprendizaje. Reflexiones Teológicas, (6), 167-195. Recuperado de https://dialnet.unirioja.es/servlet/articulo?codigo=3709190

Constitución Política de Colombia. (13 de junio de 1991). Recuperado de https://bibliotecadigital.ccb.org.co/bitstream/handle/11520/24627/CONSTITUCION\%20 POLITICA\%201991.pdf?sequence $=1$

De León, Beatriz. (2011). La relación familia-escuela y su repercusión en la autonomía y responsabilidad de los niños/as. Presentado en el XII Congreso Internacional de educación. Barcelona, España. Recuperado de https://extension.uned.es/archivos publicos/webex actividades/5385/repercusiones $\underline{\text { 8.pdf }}$

De Zubiria, Julián. (06 de junio, 2020). La educación en tiempos de cuarentena. Revista Semana. Recuperado de https://www.semana.com/opinion/articulo/la-educacion-entiempos-de-cuarentena-columna-de-julian-de-zubiria/661969/

El País. (2020, 2 de marzo). La Unesco advierte de que el cierre de escuelas por coronavirus puede aumentar las desigualdades sociales. Recuperado de https://elpais.com/sociedad/2020-03-05/la-unesco-advierte-de-que-el-cierre-deescuelas-por-coronavirus-puede-aumentar-las-desigualdades-sociales.html

Escobedo, Rubén., Pimentel, Angelina. y Cruz, Claudia. (2020). El Aprendizaje Autónomo en El Sistema a Distancia. Revista de Desarrollo Sustentable, Negocios, Emprendimiento y Educación, 2(7), 1-15. Recuperado de https://www.eumed.net/rev/rilcoDS/07/aprendizaje-autonomo.html 
Flores, José Luis. (2020). La sociedad y la comunicación desde la perspectiva de Manuel Castells de sociedad red. Sintaxis, 1(5), 85-102. Recuperado de http://revistas.anahuac.mx/sintaxis/article/view/405

García, Roberto., Caldas, Johan., Dávila, Diego. y Thoene, Ulf. (2020). Políticas públicas de inclusión digital en Colombia. Una evaluación del Plan Vive Digital (2010-2014). Revista espacios, 41(7), $1-13 . \quad$ Recuperado de http://www.revistaespacios.com/a20v41n07/20410713.html

Guadagni, Alieto Aldo. (2020). Evitemos que la pandemia global amenace nuestras escuelas. Recuperado de http://repositorio.ub.edu.ar/handle/123456789/8952

Gobierno Nacional de Colombia. (22 de marzo, 2020). Decreto 457, mediante el cual se imparten instrucciones para el cumplimiento del Aislamiento Preventivo Obligatorio. Recuperado de https://cutt.ly/DWhWl1y

Johnson, María Cecilia., Saletti-Cuesta, Lorena. y Tumas, Natalia. (2020). Emociones, preocupaciones y reflexiones frente a la pandemia del COVID-19 en Argentina. Ciência \& Saúde Coletiva, 25(1), 2447-2456. Recuperado de https://www.scielo.br/scielo.php?script=sci arttext\&pid=S1413$\underline{81232020006702447}$

Ley 115. Ley por la cual se expide la Ley General de la Educación. (1994). Recuperado de http://www.secretariasenado.gov.co/senado/basedoc/ley 0115 1994.html

Ley 1176. Ley por la cual se dictan normas orgánicas dictan normas para el Sistema General de Participaciones (SIGEP). (2007). Recuperado de http://www.secretariasenado.gov.co/senado/basedoc/ley 1176 2007.html

Lorente, Sandra. (2018). Revisión de los planes de igualdad vigentes en las universidades públicas de Cataluña (Tesis de Maestría). Universidad Jaume I Andalucía, España. Recuperado de http://repositori.uji.es/xmlui/bitstream/handle/10234/180826/TFM 2018 GraciaLorente Sandra.pdf?sequence $=1$

Martínez, Raúl. y Sánchez, Galo. (2020). El Aprendizaje Cooperativo en la clase de Educación Física: dificultades iniciales y propuestas para su desarrollo. Revista Educación,44(1).

Recuperado de https://www.scielo.sa.cr/pdf/edu/v44n1/2215-2644-edu-44-01-00019.pdf

Mcluhan, Marshall. y Powers, Bruce. (1989). La aldea global. Transformaciones en la vida y los medios de comunicación mundiales en el siglo XXI. Barcelona, España: Gedisa. Recuperado de https://cutt.ly/QWhWp4J

Ministerio de Educación Nacional (MEN). (2016). Revisión de políticas nacionales de educación. La educación en Colombia. Recuperado de https://www.mineducacion.gov.co/1759/articles-356787 recurso 1.pdf 
Ministerio de Educación Nacional (MEN). (2016). Revolución educativa. Programa para el desarrollo competencias.

Recuperado de https://www.mineducacion.gov.co/1759/articles-

217596 archivo pdf desarrollocompetencias.pdf

Ministerio de Educación Nacional (MEN). (2020a, 16 de marzo). Circular No. 20 del 16 de marzo de 2020: Medidas adicionales y complementarias para el manejo, control y prevención del Coronavirus (COVID-19). Recuperado de https://www.mineducacion.gov.co/1759/w3-article-394018.html? noredirect=1

Ministerio de Educación Nacional (MEN). (2020b, 22 de marzo). Juntos para existir, convivir y aprender. Recuperado de https://www.mineducacion.gov.co/1759/articles394577 recurso 4. pdf

Ministerio de las Tecnologías de la comunicación y la información [MINTIC]. (2019). Conectar a Colombia: un reto que cambiará vidas. Recuperado de https://www.mintic.gov.co/portal/inicio/Sala-de-Prensa/Columnas-MinistraTIC/98249:Conectar-a-Colombia-un-reto-que-cambiara-vidas

Organización de la Naciones Unidas para la Educación, la Ciencia y la cultura (Unesco). (2020). La educación en tiempos de la pandemia de COVID-19. Recuperado de https://repositorio.cepal.org/bitstream/handle/11362/45904/S2000510 es.pdf?sequence $=1$ \&isAllowed $=y$

Organización para la Cooperación y el Desarrollo Económicos (OCDE). (2020). Un marco para guiar una respuesta educativa a la pandemia del 2020 del COVID-19. Recuperado de https://fe.ccoo.es/30b7657046638d44d2a86dcc6a602045000063.pdf

Peinado, José de Jesús. (2020). Experiencias del profesorado acerca del aprendizaje autónomo en estudiantes de modalidad a distancia y el uso de recursos digitales. Revista Iberoamericana para la Investigación y el Desarrollo Educativo, 10(20), 1-17. Recuperado de http://www.scielo.org.mx/scielo.php?pid=S2007$\underline{74672020000100130 \& \text { script }=\text { sci } \text { arttext }}$

Restrepo, Johan. y Gómez Dany. (2020). La Conectividad Digital como Derecho Fundamental en Colombia. The Law, State and Telecommunications Review, Brasilia, 12(1), 113136. Doi https://doi.org/10.26512/lstr.v12i1.31161

Sánchez, Melcho.r, Martínez, Ana María del Pilar., Torres, Ruth., de Agüero Servín, Mercedes., Hernández Alan., Benavides, Mario., Rendón, Víctor. y Jaimes, Carlos. (2020). Retos educativos durante la pandemia de covid-19: una encuesta a profesores de la UNAM. Revista Digital Universitaria, 21(3), $1-24 . \quad$ doi: http://doi.org/10.22201/codeic.16076079e.2020.v21n3.a12

Simón, Cecilia. y Barrios, Angela. (2019). Las familias en el corazón de la educación inclusiva. Aula Abierta, 48(1), 51-58. Recuperado dehttps://dialnet.unirioja.es/servlet/articulo?codigo=6861533 
Sistema Económico Latinoamericano y del Caribe (SELA). (2021). La conectividad digital Rural: Un desafío clave para el desarrollo de América Latina y el Caribe. Recuperado dehttp://www.sela.org/es/eventos/e/71599/conectividad-digital-rural

Solorzano, Yelena. (2017). Aprendizaje autónomo y competencias. Dominio de las ciencias, 3(1), 241-253. Recuperado de https://dialnet.unirioja.es/servlet/articulo?codigo=5907382

Solovieva, Yulia. y Quintanar, Luis. (2020). Efectos del confinamiento por la pandemia COVID2020 sobre el proceso de enseñanza-aprendizaje en los niveles básico y medio en el Estado de Puebla. Recuperado de http://www.concytep.gob.mx/wpcontent/uploads/2020/05/efectos del confinamiento por la pandemia.pdf

Tonucci, Francisco. (2020, 4 de junio). Solo los buenos maestros podrán salvar la escuela. Revista digital Educación 3.0. Recuperado de https://www.educaciontrespuntocero.com/entrevistas/francesco-tonucci-entrevista/

Vijil, Josefina. y Castillo, Melba. (2020). Estamos ante una emergencia educativa, en la educación nos estamos jugando el presente y el futuro. Revista Envío,456(52), 10-25. Recuperado de https://www.envio.org.ni/articulo/5747 
Revista indizada en

sciplo redalyc sy latindex DOAJ J

Distribuida en las bases de datos:

- Dialnet

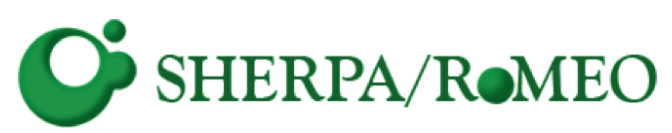

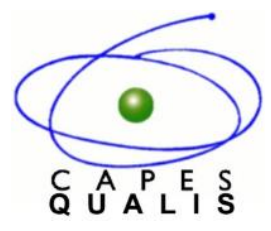

MIAR 